\title{
WASP-21b: a hot-Saturn exoplanet transiting a thick disc star ${ }^{\star} \star \star$
}

\author{
F. Bouchy ${ }^{1,2}$, L. Hebb ${ }^{3,5}$, I. Skillen ${ }^{4}$, A. Collier Cameron ${ }^{5}$, B. Smalley ${ }^{6}$, S. Udry ${ }^{7}$, D. R. Anderson ${ }^{6}$, I. Boisse ${ }^{1}$, \\ B. Enoch ${ }^{5}$, C. A. Haswell ${ }^{8}$, G. Hébrard ${ }^{1}$, C. Hellier ${ }^{6}$, Y. Joshi ${ }^{9}$, S. R. Kane ${ }^{10}$, P. F. L. Maxted ${ }^{6}$, M. Mayor ${ }^{7}$, \\ C. Moutou ${ }^{11}$, F. Pepe ${ }^{7}$, D. Pollacco ${ }^{9}$, D. Queloz ${ }^{7}$, D. Ségransan ${ }^{7}$, E. K. Simpson ${ }^{9}$, A. M. S. Smith ${ }^{6}$, H. C. Stempels ${ }^{12}$, \\ R. Street ${ }^{13}$, A. H. M. J. Triaud ${ }^{7}$, R. G. West ${ }^{14}$, and P. J. Wheatley ${ }^{15}$

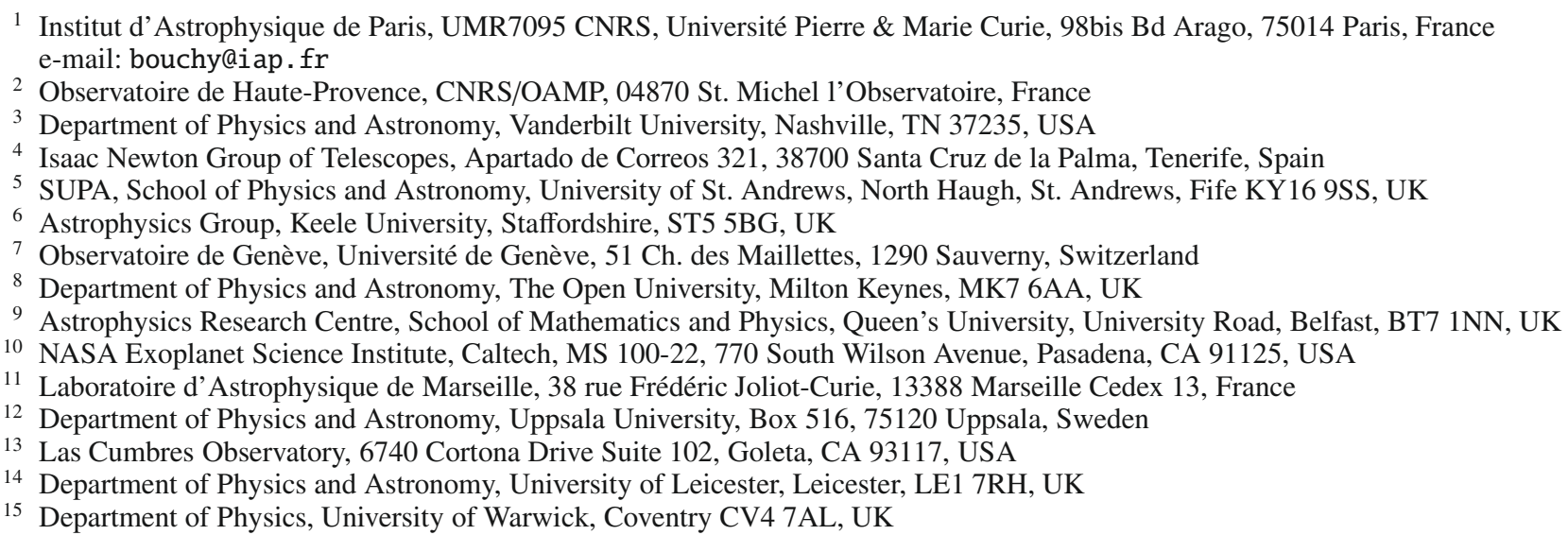

Received 19 April 2010 / Accepted 13 May 2010

\begin{abstract}
We report the discovery of WASP-21b, a new transiting exoplanet discovered by the Wide Angle Search for Planets (WASP) Consortium and established and characterized with the FIES, SOPHIE, CORALIE and HARPS fiber-fed echelle spectrographs. A 4.3-d period, 1.1\% transit depth and 3.4-h duration are derived for WASP-21b using SuperWASP-North and high precision photometric observations at the Liverpool Telescope. Simultaneous fitting to the photometric and radial velocity data with a Markov Chain Monte Carlo procedure leads to a planet in the mass regime of Saturn. With a radius of $1.07 R_{\mathrm{Jup}}$ and mass of $0.30 M_{\mathrm{Jup}}$, WASP-21b has a density close to $0.24 \rho_{\text {Jup }}$ corresponding to the distribution peak at low density of transiting gaseous giant planets. With a host star metallicity $[\mathrm{Fe} / \mathrm{H}]$ of -0.46 , WASP-21b strengthens the correlation between planetary density and host star metallicity for the five known Saturn-like transiting planets. Furthermore there are clear indications that WASP-21b is the first transiting planet belonging to the thick disc.
\end{abstract}

Key words. planetary systems - techniques: photometric - techniques: radial velocities

\section{Introduction}

Observations of planets that transit their host star represent currently the best opportunity to test models of exoplanet structure and evolution. These last ten years, the photometric surveys have led to an increasing list of transiting planets. More than seventy transiting planetary systems have been identified from

\footnotetext{
* Based on observations made with the SuperWASP-North camera hosted by the Isaac Newton Group on La Palma, the FIES spectrograph on the Nordic Optical Telescope, the CORALIE spectrograph on the 1.2-m Euler Swiss telescope on La Silla Observatory, the SOPHIE spectrograph on the 1.93-m telescope on Haute Provence Observatory and the HARPS spectrograph on the 3.6-m ESO telescope at La Silla Observatory under programs 081.C-0388, 082.C-0040, 084.C-0185.

$\star \star$ Tables of photometric data are only available in electronic form at the CDS via anonymous ftp to cdsarc.u-strasbg.fr (130.79.128.5) or via

http://cdsarc.u-strasbg.fr/viz-bin/qcat?J/A+A/519/A98
}

Super-Earth to Jupiter-like planets. The WASP project operates two identical instruments, at La Palma in the Northern hemisphere, and at Sutherland in South Africa in the Southern hemisphere and led recently to the detection of about $30 \%$ of known transiting planets. Each WASP telescope has a field of view of just under 500 square degrees. The WASP survey is sensitive to planetary transit signatures in the light curves of stars in the magnitude range $V \sim 9-13$. A detailed description of the telescope hardware, observing strategy and pipeline data analysis is given in Pollacco et al. (2006).

Here we present the WASP photometry of SWASPJ230958.23+182346.0, high-precision photometric follow-up observations with the RISE instrument on the Liverpool Telescope and high-precision radial velocity (RV) observations with the FIES (2.6-m NOT), SOPHIE (1.93-m OHP), CORALIE (1.2-m Euler) and HARPS (3.6-m ESO) fiber-fed echelle spectrographs. These observations lead to the discovery of WASP-21b, a hot-Saturn transiting exoplanet. 


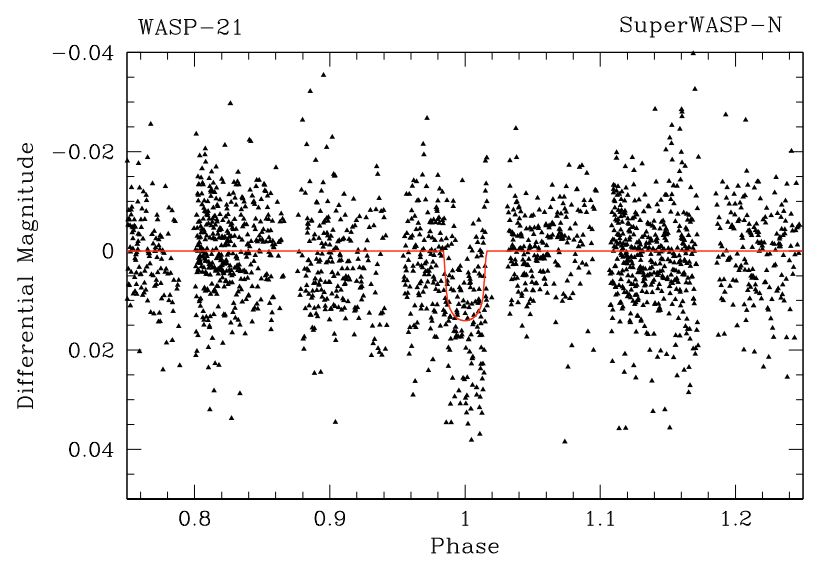

Fig. 1. Un-binned SuperWasp folded light curve for WASP-21 with the ephemeris $P=4.32$ days and $T_{0}=2454743.0419$.

\section{Observations}

\subsection{SuperWASP observation}

SuperWASP-North is a multi-camera telescope system located in La Palma and consisting of 8 Canon 200-mm $f / 1.8$ lenses each coupled to e2v $2048 \times 2048$ pixel back illuminated CCDs (Pollacco et al. 2006). This combination of lens and camera yields a field-of-view of $7.8 \times 7^{\circ} .8$ with an angular size of 13.7 arcsec per pixel. The target SWASPJ230958.23+182346.0 was monitored from 2006 July 22 to November 27, totaling 3814 photometric measurements (available in electronic form at CDS). The pipeline-processed data were detrended and searched for transits with the methods described in Collier Cameron et al. (2006), yielding a detection of a periodic transitlike signature with a period of 4.32 days. Figure 1 shows the unbinned SuperWASP phase-folded light curve of WASP-21 with the ephemeris $P=4.32$ days and $T_{0}=2454743.0419$.

\subsection{Higher precision photometric follow-up}

On-off photometric observations were made with the 2-m Faulkes Telescope North (FTN) at Hawaii on 2th September 2008 with the Pan-STARRS-z broad-band filter. These observations allowed the transit signal to be confirmed. Follow-up photometry was performed with the fast read-out camera RISE located on the 2.0-m Liverpool Telescope at La Palma. Although the night was non-photometric and the sky was variable, a partial transit was observed on 7th October 2008 in agreement with the SuperWasp and FTN photometry. The phase-folded light curve of FTN and RISE (available in electronic form at CDS) are shown in Fig. 2.

On October 2009, out-of-transit high-angular resolution images were provided on the 4.2-m WHT with the Adaptive Optic NAOMI and the IR imager INGRID. Ks-band images have a full width at half maximum (FWHM) of 0.18 arcsec with circular contours, excluding evidence of a visible companion on that spatial scale. The limit in magnitude is estimated to be $K \mathrm{~s} \sim 13.2$ at 0.36 arcsec and to be $K s \sim 16.8$ away from the tail of the point spread function (PSF).

\subsection{Doppler follow-up}

Three spectra were first obtained at the end of December 2007 with the FIES spectrograph at the 2.6-m Nordic Optical

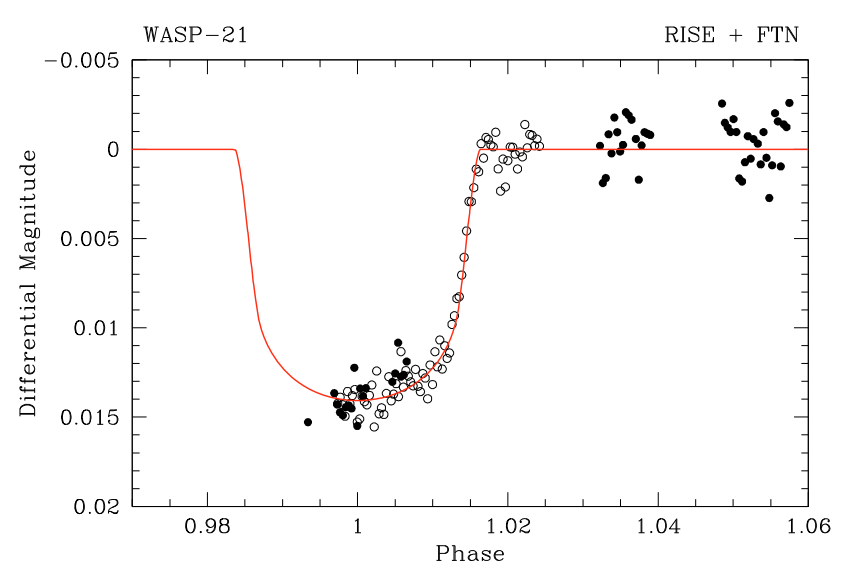

Fig. 2. Phase-folded light curve from the RISE (open circle) and FTN (black circle) photometry for WASP-21 superposed with the MCMC solutions. The RISE photometry was binned 2-min in time.

Telescope (NOT - La Palma). The combined spectrum indicated a dwarf star and the radial velocities showed no variations greater than $100 \mathrm{~m} \mathrm{~s}^{-1}$, excluding a low-mass star companion. Radial velocity (RV) follow-up of WASP-21 was conducted with the echelle spectrograph SOPHIE at the 1.93-m telescope of Observatoire de Haute Provence (Perruchot et al. 2008; Bouchy et al. 2009), CORALIE at the 1.2-m Swiss Euler telescope of La Silla (Baranne et al. 1996; Queloz et al. 2000; Pepe et al. 2002), and HARPS at the 3.6-m telescope of ESO La Silla (Mayor et al. 2003). A total of eleven SOPHIE measurements were made in July and August 2008 with about the same signalto-noise ratio $S / N=30$ in order to minimize the charge transfer inefficiency (CTI) effect (Bouchy et al. 2009) although it is now corrected by the data reduction software (DRS). Some measurements were made with moon light but without significant effect on the RV measurements considering that the RV of the target $\left(-89.4 \mathrm{~km} \mathrm{~s}^{-1}\right)$ was always far from the moon RV. The RV uncertainties were computed assuming photon noise plus $15 \mathrm{~m} \mathrm{~s}^{-1}$ quadratically added in order to take into account the systematics of the high efficiency mode like the guiding and centering errors (Boisse et al. 2010) and wavelength calibration uncertainty. Five CORALIE measurements were made simultaneously with those of SOPHIE and eight additional ones were made one year later in June and September 2009. One CORALIE point was made close to the transit epoch (BJD $=2454686.83$ ) and may be affected by the Rossiter-McLaughlin effect. We did not use it to determine the Keplerian solution. However, considering the small $v \sin i\left(\leq 2 \mathrm{~km} \mathrm{~s}^{-1}\right)$ of the star, the expected amplitude of the RM effect should be lower than $20 \mathrm{~m} \mathrm{~s}^{-1}$, which makes it difficult to detect with CORALIE or SOPHIE. Nine HARPS measurements were made in September and October 2008 to definitively secure the candidate considering the small RV amplitude observed by SOPHIE and CORALIE, and also to exclude blended eclipsing binaries scenario and to provide a high $S / N$ spectrum for stellar parameters analysis (see Sect. 3.1). Six HARPS measurements were added in October 2009 to check for eccentricity and for additional companion in the system.

The phase-folded RVs in Fig. 3 shows the SOPHIE (black circles), HARPS (red squares), CORALIE (green triangles) and FIES (blue open circles) points. All are phase matched pretty well with the photometry. The best Keplerian fit was obtained with an RV offset from the HARPS data of +11 , -20.5 and $+15 \mathrm{~m} \mathrm{~s}^{-1}$ for the CORALIE, SOPHIE and FIES 
Table 1. Radial velocities of WASP-21.

\begin{tabular}{llc}
\hline \hline \multicolumn{1}{c}{ BJD } & $\begin{array}{c}\mathrm{RV} \\
-2400000\end{array}$ & $\begin{array}{c} \pm 1 \sigma \\
\left(\mathrm{km} \mathrm{s}^{-1}\right)\end{array}$ \\
$\left(\mathrm{km} \mathrm{s}^{-1}\right)$
\end{tabular}

data respectively. The semi-amplitude of the RV curve is $K=$ $37.2 \pm 1.1 \mathrm{~m} \mathrm{~s}^{-1}$. We adjust the Keplerian orbit fixing the eccentricity to zero. There is no obvious evidence for eccentricity, although it is difficult to fit for the eccentricity with a small amount of data points from four different instruments because we already have three other free parameters (the zero-point offsets of the RV data sets).

No significant RV drift was detected with HARPS during one year excluding additional Jupiter-like companion with a period of less than one year.

Figure 4 shows the bisector span versus RV. The SOPHIE and CORALIE bisector spans have too large uncertainties to exclude a possible correlation with radial velocities. The HARPS bisector spans definitively show no correlation, and scenarios of blended eclipsing binaries are definitively invalided.

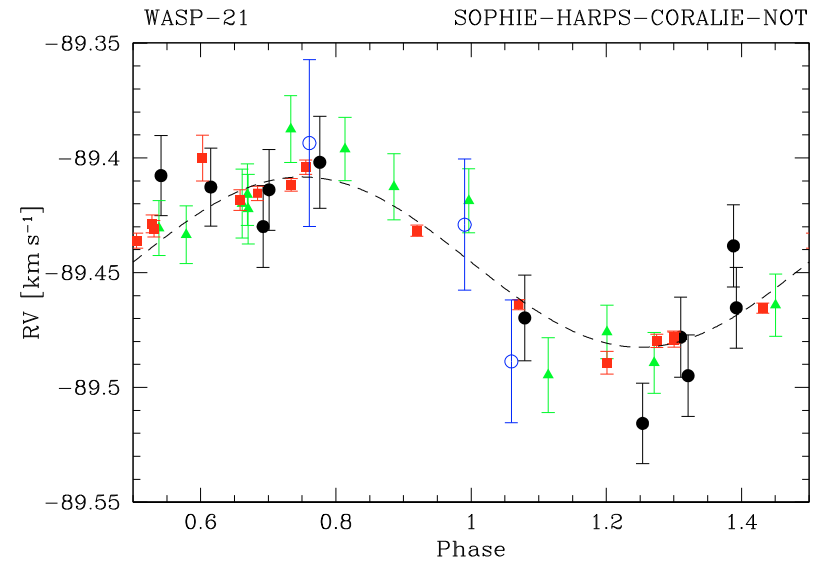

Fig. 3. Phase-folded radial velocities of WASP-21 obtained with SOPHIE (black circles), HARPS (red squares), CORALIE (green triangles) and FIES (blue open circles).

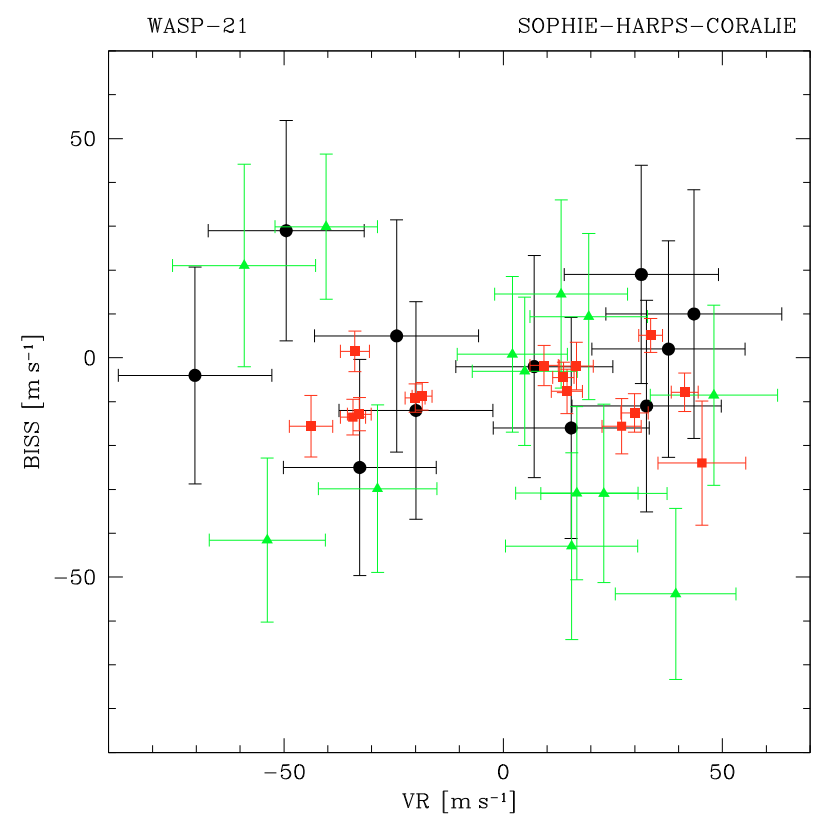

Fig. 4. Bisector span versus radial velocities of WASP-21 obtained with SOPHIE (black circles), HARPS (red squares) and CORALIE (green triangles) without significant correlation and invalidating scenarios of blended eclipsing binaries.

\section{Results and parameters}

\subsection{Stellar parameters}

A detailed spectroscopic analysis of the stellar atmospheric properties was made with the FIES and HARPS spectra. We merged the HARPS spectra into one high-quality spectrum in order to perform a detailed spectroscopic analysis of the stellar atmospheric properties. This merged spectrum was continuumnormalized with a low-order polynomial to retain the shape of the broadest spectral features. The total signal-to-noise ratio of the combined spectrum was about 100 per pixel.

As previously done for our analysis of WASP-1 (Stempels et al. 2007) and WASP-3 (Pollacco et al. 2008), we employed the methodology of Valenti \& Fischer (2005), using the same tools, techniques and model atmosphere grid. We used the package Spectroscopy Made Easy (SME) (Valenti \& Piskunov 1996), 
Table 2. Stellar parameters for WASP-21 derived from FIES and HARPS spectroscopy.

\begin{tabular}{lc}
\hline \hline Parameter & WASP-21 \\
\hline GSC & $01715-00679$ \\
2MASS & J23095825+1823459 \\
RA $(J 2000)$ & $23: 09: 58.25$ \\
Dec $(J 2000)$ & $+18: 23: 45.9$ \\
$V$ & $11.58 \pm 0.08$ \\
Distance & $242_{-30}^{+50} \mathrm{pc}$ \\
$T_{\text {eff }}$ & $5800 \pm 100 \mathrm{~K}$ \\
$\log g$ & $4.2 \pm 0.1$ \\
{$[\mathrm{M} / \mathrm{H}]$} & $-0.4 \pm 0.1$ \\
$v \sin i$ & $1.5 \pm 0.6 \mathrm{~km} \mathrm{~s}^{-1}$ \\
$v_{\mathrm{rad}}$ & $-89.45 \mathrm{~km} \mathrm{~s}$ \\
$\mathrm{Age}$ & $12 \pm 5 \mathrm{Gyr}$ \\
\hline $\log A(\mathrm{Li})$ & $2.19 \pm 0.09$ \\
{$[\mathrm{Na} / \mathrm{H}]$} & $-0.47 \pm 0.11$ \\
{$[\mathrm{Mg} / \mathrm{H}]$} & $-0.28 \pm 0.08$ \\
{$[\mathrm{Ca} / \mathrm{H}]$} & $-0.25 \pm 0.13$ \\
{$[\mathrm{Ti} / \mathrm{H}]$} & $-0.28 \pm 0.10$ \\
{$[\mathrm{Mn} / \mathrm{H}]$} & $-0.75 \pm 0.14$ \\
{$[\mathrm{Fe} / \mathrm{H}]$} & $-0.46 \pm 0.11$ \\
{$[\mathrm{Si} / \mathrm{H}]$} & $-0.25 \pm 0.12$ \\
{$[\mathrm{Sc} / \mathrm{H}]$} & $-0.33 \pm 0.11$ \\
{$[\mathrm{~V} / \mathrm{H}]$} & $-0.32 \pm 0.10$ \\
{$[\mathrm{Cr} / \mathrm{H}]$} & $-0.44 \pm 0.17$ \\
{$[\mathrm{Co} / \mathrm{H}]$} & $-0.39 \pm 0.14$ \\
{$[\mathrm{Ni} / \mathrm{H}]$} & $-0.46 \pm 0.14$ \\
\hline
\end{tabular}

which combines spectral synthesis with multidimensional $\chi^{2}$ minimization to determine which atmospheric parameters best reproduce the observed spectrum of WASP-21 (effective temperature $T_{\text {eff }}$, surface gravity $\log g$, metallicity $[\mathrm{M} / \mathrm{H}]$, projected $v \sin i$, microturbulence $v \_$mic and the macroturbulence $\left.v \_m a c\right)$. The parameters we obtained from this analysis are listed in Table 2.

We interpolated the Girardi evolutionary tracks using a 12 Gyr old star with $T_{\text {eff }}=5800$ and $[\mathrm{M} / \mathrm{H}]=-0.4$ and found $M v=4.97$. A reddening $E(B-V)$ of 0.1 was estimated from the equivalent width of interstellar $\mathrm{NaD}$ lines with the Munari \& Zwitter (1997) relationship. With an observed $V$ mag of 11.58 , the distance is estimated to be $242_{-30}^{+50} \mathrm{pc}$. This value agrees with the distance of $235 \mathrm{pc}$ derived through the angular diameter ( $\theta=0.042 \pm 0.002$ mas) estimated from the infrared flux method and the stellar radius from Table 3 . It also agrees with the distance of $230 \pm 30 \mathrm{pc}$ based on the measured $V$ band magnitude $(V=11.58 \pm 0.08)$ and the absolute $V$ magnitude of a typical G3V type star (Gray 1988). The properties of WASP-21 suggest it is a member of the thick disc component of the Galaxy. With the NOMAD catalogue proper motions (Zacharias et al. 2004) and the measured systemic radial velocity, we calculated its $U$, $V$, and $W$ space motion to be $-34.6,-66.0,+62.1 \mathrm{~km} \mathrm{~s}^{-1}$ relative to the Sun, adopting a distance of $242 \mathrm{pc}$ based on the measured $V$ band magnitude $(V=11.58 \pm 0.08)$ and the absolute $V$ magnitude of a typical G3V type star (Gray 1988). It is clear that WASP-21 is lagging behind the Sun (which moves with the thin disc) with a high negative $V$ velocity similar to the canonical thick disc value of $\sim-45 \mathrm{~km} \mathrm{~s}^{-1}$ (Morrison et al. 1990). WASP21 is also metal-poor with $[\mathrm{M} / \mathrm{H}]=-0.4 \pm 0.1$, again similar to the mean thick disc value of $\sim-0.6$ dex. In addition, the abundance analysis shows enhanced $\alpha / \mathrm{Fe}$ ratios, which is also typical of a thick disc star (see Edvardsson et al. 1993). The alpha elements, $\mathrm{Mg} \mathrm{Ca}, \mathrm{Ti}$, and $\mathrm{Si}$ have abundances of $\sim-0.25$, approximately twice the value of iron, $[\mathrm{Fe} / \mathrm{H}]=-0.46$, and the
Table 3. WASP-21 system parameters and $1 \sigma$ error limits derived from the MCMC analysis.

\begin{tabular}{lc}
\hline \hline Parameter & Value \\
\hline Transit epoch $T_{0}[\mathrm{HJD}]$ & $2454743.0419_{-0.0022}^{+0.0019}$ \\
Orbital period $P$ [days] & $4.322482_{-0.000024}^{+0.00019}$ \\
Planet/star area ratio $\left(R_{\mathrm{p}} / R_{*}\right)^{2}$ & $0.01082_{-0.003035}^{+0.0037}$ \\
Transit duration $t_{\mathrm{T}}[$ days $]$ & $0.1398_{-0.0040}^{+0.0088}$ \\
Impact parameter $b\left[R_{*}\right]$ & $0.23_{-0.15}^{+0.12}$ \\
Orbital inclination $I$ [degrees] & $88.75_{-0.70}^{+0.84}$ \\
& \\
Stellar reflex velocity K $\left[\mathrm{m} \mathrm{s}{ }^{-1}\right]$ & $37.2 \pm 1.1$ \\
Orbital semimajor axis $a[\mathrm{AU}]$ & $0.052_{-0.00044}^{+0.00041}$ \\
& \\
Stellar mass $M_{*}\left[M_{\odot}\right]$ & $1.01 \pm 0.03$ \\
Stellar radius $R_{*}\left[R_{\odot}\right]$ & $1.06 \pm 0.04$ \\
Stellar surface gravity log $g_{*}[\mathrm{cgs}]$ & $4.39 \pm 0.03$ \\
Stellar density $\rho_{*}\left[\rho_{\odot}\right]$ & $0.84 \pm 0.09$ \\
& \\
Planet mass $M_{\mathrm{p}}\left[M_{\mathrm{J}}\right]$ & $0.300 \pm 0.011$ \\
Planet radius $R_{\mathrm{p}}\left[R_{\mathrm{J}}\right]$ & $1.07 \pm 0.06$ \\
Planet density $\rho_{\mathrm{p}}\left[\rho_{\mathrm{J}}\right]$ & $0.24 \pm 0.05$ \\
\hline
\end{tabular}

other iron peak elements $([\mathrm{V} / \mathrm{H}]=-0.32,[\mathrm{Cr} / \mathrm{H}]=-0.44$, $[\mathrm{Mn} / \mathrm{H}]=-0.75,[\mathrm{Co} / \mathrm{H}]=-0.39$ and $[\mathrm{Ni} / \mathrm{H}]=-0.46)$. Furthermore, when we compare the effective temperature $\left(T_{\text {eff }}=\right.$ $5800 \pm 100 \mathrm{~K})$, metallicity and mean stellar density $\left(\rho / \rho_{\odot}=0.84\right)$ derived from the transit parameters of WASP-21 to theoretical stellar evolution models from Girardi et al. (2000) as described in Hebb et al. (2009), we find the star to be evolved off the zero-age main sequence and similar in age to that of the thick disc (age $\sim 12 \pm 5 \mathrm{Gyr}$ ). In order to quantify the probability that WASP-21 is a member of the thick disc, we compared its chemical and kinematic properties to the properties of similar model stars in the Besançon Galactic model (Robin et al. 2003) where the population class of the stars are known. We selected a set of model main sequence stars in a small volume nearby the position of WASP-21 for comparison. We generated more than 800000 model stars in 55 realizations of the simulation with spectral types of F5V-K7V, distances of 150-600 pc, and positions within $\pm 5^{\circ}$ in each direction of WASP-21 $(l=92$, $b=-38)$. The model stars come from all Galactic components and are not selected based on their velocities or metallicities. Of the model stars with metallicities between -0.5 and -0.3 dex and within $\pm 15 \mathrm{~km} \mathrm{~s}^{-1}$ of the calculated $U, V$, and $W$ values of WASP-21, all have ages $\geq 7 \mathrm{Gyr}$ (population class 7 and 8) which suggests WASP-21 is old. Furthermore, $92 \%$ of the matching model stars belong to the thick disc. In summary, WASP-21 has a high probability of being a thick disc member based on its kinematic properties, low metallicity, abundance patterns, and likely old age.

\subsection{Planet parameters}

To determine the planetary and orbital parameters the radial velocity measurements were combined with the photometry from WASP, RISE and FTN in a simultaneous fit with the Markov Chain Monte Carlo (MCMC) technique. The details of this process are described in Pollacco et al. (2008). Recent features and improvements were included. The linear de-trending of the optical light curves was made with respect to phase at each step in the MCMC chain. An initial fit showed that the orbital eccentricity was poorly constrained by the available data and nearly consistent with zero. We therefore fixed the eccentricity parameter 
at zero. We derived the host star mass consistently within the MCMC code by comparing the mean stellar density (measured from the shape of the transit) and the observed metallicity and effective temperature of the host star to an empirical relation defined by a set of detached eclipsing binaries with component masses and radii measured to high precision $(\leq 3 \%)$ (Torres et al. 2010). With this novel technique, we derived a mass for WASP21 of $M=1.01 \pm 0.03 M_{\odot}$. The mass and radius of WASP$21 \mathrm{~b}$, given in Table 3 with the other best-fit parameters, are then found to be $M_{P}=0.300 \pm 0.011 M_{\mathrm{Jup}}$ and $R_{P}=1.07 \pm 0.06 R_{\mathrm{Jup}}$, respectively.

Finally, we note that the host star mass derived from the theoretical stellar evolution models of Girardi et al. (2000) is significantly less than what we find using the empirical relation, even though we used the same observed stellar density, temperature and metallicity. Upon further investigation, we found that the metallicity has a strong effect on the radii and effective temperatures of the theoretical stars, which is not present in the observed eclipsing binary systems. Specifically, a $\sim 5800 \mathrm{~K}$, solar metallicity $(Z=0.019)$ theoretical star with an age of $5 \mathrm{Gyr}$ has a predicted mass of $1.0 M_{\odot}$, but at $Z=0.008([\mathrm{M} / \mathrm{H}]=-0.37)$, the predicted mass is $\sim 10 \%$ less $\left(0.9 M_{\odot}\right)$. However, the four eclipsing binary components in Torres et al. (2010) with temperatures within $\pm 100 \mathrm{~K}$ of WASP-21, have $[\mathrm{Fe} / \mathrm{H}]$ measurements ranging from -0.1 to +0.24 , but masses similar to within $5 \%$. There is a clear difference between the predicted effects of metallicity on the properties of stars and the observed effects in eclipsing binaries. Using a stellar mass of $0.9 M_{\odot}$ leads to a planetary mass and radius for WASP-21b of $M_{P}=0.29 \pm 0.01 M_{\text {Jup }}$ and $R_{P}=1.03 \pm 0.06 R_{\mathrm{Jup}}$, respectively, $\sim 3 \%$ less than our adopted value.

\section{Discussion and conclusion}

Figure 5 shows the mass-radius diagram of known gas-giant transiting exoplanets (with a mass of more than $0.1 M_{\text {Jup }}$ ). WASP-21b is one of the only five transiting gas-giant planets with a mass below $0.4 M_{\text {Jup }}$ including HAT-P-12b (Hartman et al. 2009), HD 149026b (Sato et al. 2005), CoRoT-8b (Bordé et al. 2010) and WASP-29b (Hellier et al. 2010).

Figure 6 shows the density histogram of transiting gas-giant planets with a mass between 0.2 and 1.5 Jupiter masses (totaling 50 exoplanets). The probability density function presents a clear asymmetric distribution peaking at the lowest densities $\left(0.25 \rho_{\text {Jup }}\right)$ and decreasing from 0.25 to $1.25 \rho_{\text {Jup }}$. WASP-21b and HAT-P-12b, both with a density of $\sim 0.24 \rho_{\text {Jup }}$, are bloated planets with a density close to the mode of that distribution. This distribution is biased because the transit detection method (especially from ground) favours planets with large radii, hence with lower density for a given mass. If large radii are predominantly the result of irradiation, the selection effects will favour stars with small $a / R_{*}$, because that gives a stronger irradiation of the planet. Small $a / R_{*}$ also increases the transit detection probability.

Only four transiting exoplanets with a mass in the range $0.2-1.5 M_{\text {Jup }}$ have a density greater than or equal to Jupiter including the two Saturn-like planets CoRoT-8b and HD 149026b as well as WASP-7b (Hellier et al. 2008) and OGLE-TR-113b (Bouchy et al. 2004), all of them having a host star with metallicity $[\mathrm{Fe} / \mathrm{H}] \geq 0$. The density of the five Saturn-like transiting planets appears to be clearly correlated with the metallicity of their host stars $([\mathrm{Fe} / \mathrm{H}]=-0.46,-0.29,+0.11,+0.3$ and +0.36 for WASP-21b and HAT-P-12b, WASP-29b, CoRoT-8b and HD $149026 \mathrm{~b}$ respectively), confirming and reinforcing the

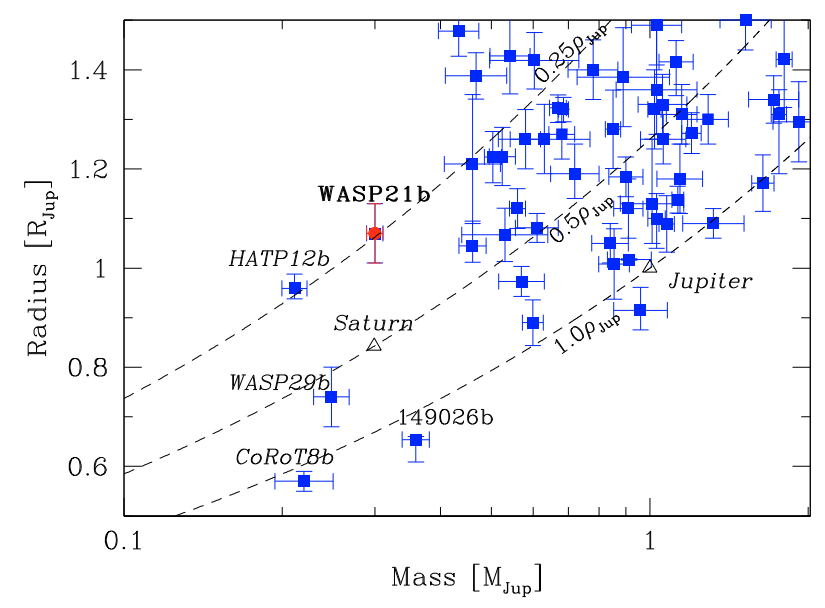

Fig. 5. Mass-radius diagram of transiting gaseous giant exoplanets $\left(M_{\mathrm{P}} \geq 0.1 M_{\mathrm{Jup}}\right)$.

relation established by Guillot et al. (2006). If low host star metallicity also favours larger planetary radii, it may explain the difference observed between the host star metallicity distribution from radial velocity surveys $(50 \%$ with $[\mathrm{Fe} / \mathrm{H}] \geq 0.1)$ and transit surveys $(40 \%$ with $[\mathrm{Fe} / \mathrm{H}] \geq 0.1)$.

It is clear from previous studies that thick disc stars do form planets, which means that planet formation was going on when the Milky Way was still relatively young (i.e. as early as $\sim 12 \mathrm{Gyr}$ ago) and that these planetary systems have survived the process (i.e. major merger with a satellite galaxy) that formed the thick disc. WASP-21b is the first transiting planet with a measured radius and definitive mass to orbit a member of this population.

Reid et al. (2007) identify five previously known exoplanet host stars, which are likely thick disc members based on their subsolar metallicities, relatively large space motions (compared to the Sun) and high $[\alpha / \mathrm{Fe}]$ ratios. More recently, Neves et al. (2009) identified five planet-host stars from the 29 stars belonging to the thick disc from the HARPS GTO planet search programme indicating that approximately $17 \%$ of the thick disc stars are planet hosts. They found some indication that metalpoor $([\mathrm{Fe} / \mathrm{H}] \leq-0.2)$ planet-host stars originate preferentially in the thick disc, as also suggested by Haywood (2008). This indication is however based on small number statistics and was contested by a new spectroscopic analysis made by Gonzalez (2009), showing that according to the mass abundance of the refractory elements $(\mathrm{Mg}, \mathrm{Si}, \mathrm{Fe})$, which is important for planet formation, thick disc and $[\mathrm{Fe} / \mathrm{H}]$-poor thin disc planetary host stars have similar distributions. This author recommends to use a socalled refactory index [Ref] rather than $[\mathrm{Fe} / \mathrm{H}]$ to compare the statistics of planets around thin disc and thick disc stars. Because thick disk stars are alpha-enhanced, they might have less Fe than thin disk stars, but they have relatively more $\mathrm{Mg}$ and $\mathrm{Si}$, so they are not as metal -poor in terms of their ability to form planets as they seem to be at first glance. For WASP-21b, this refractory index [Ref] is -0.35 .

Acknowledgements. The WASP Consortium comprises astronomers primarily from the Universities of Keele, Leicester, The Open University, Queen's University Belfast, the University of St. Andrews, the Isaac Newton Group (La Palma), the Instituto de Astrofisica de Canarias (Tenerife) and the South African Astronomical Observatory. The SuperWASP-N camera is hosted by the Isaac Newton Group on La Palma with funding from the UK Science and Technology Facilities Council. We extend our thanks to the Director and staff of the Isaac Newton Group for their support of SuperWASP-N operations. Based in part on observations made at Observatoire de Haute Provence (CNRS), France, and on observations made with the Nordic Optical Telescope, operated on the island of La Palma jointly by Denmark, Finland, Iceland, Norway, 
A\&A 519, A98 (2010)

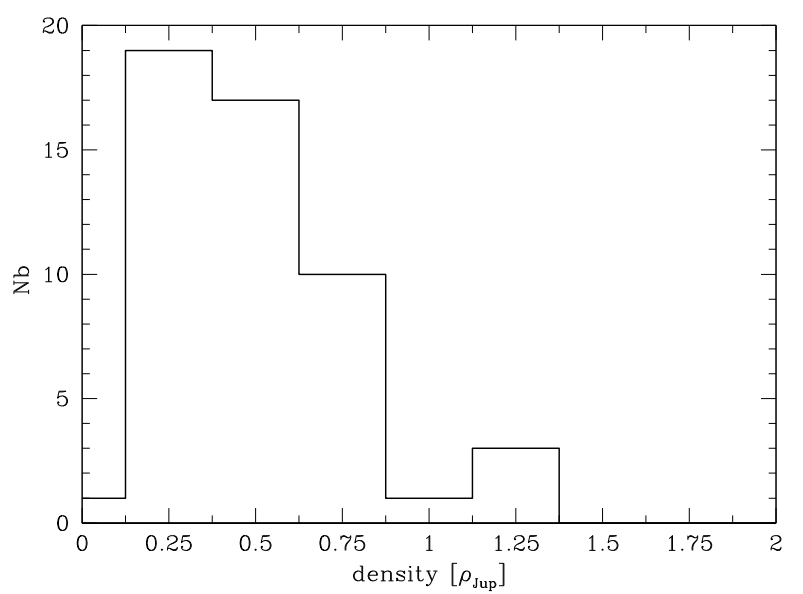

Fig. 6. Histogram of the density of transiting hot-Jupiters with a mass in the range $0.2-1.5 M_{\text {Jup }}$.

and Sweden, in the Spanish Observatorio del Roque de los Muchachos of the Instituto de Astrofisica de Canarias. We wish to thank the "Programme National de Planétologie" (PNP) of CNRS/INSU, the Swiss National Science Foundation, and the French National Research Agency (ANR-08-JCJC-0102-01) for their continuous support to our planet-search programs. F.B. would like to acknowledges P.L.S. for continuous support and advice.

\section{References}

Baranne, A., Queloz, D., Mayor, M., et al. 1996, A\&AS, 119, 373

Boisse, I., Bouchy, F., Chazelas, B., et al. 2010, in New technologies for probing the diversity of brown dwarfs and exoplanets, Shanghai, 2009, EPJ Web of Conferences, in press
Bordé, P., Bouchy, F., Deleuil, M., et al. 2010, A\&A, accepted Bouchy, F., Pont, F., Santos, N. C., et al. 2004, A\&A, 421, L13 Bouchy, F., Hébrard, G., Udry, S., et al. 2009, A\&A, 505, 853

Collier Cameron, A., Pollacco, D., Street, R. A., et al. 2006, MNRAS, 373, 799

Edvardsson, B., Andersen, J., Gustafsson, B., et al. 1993, A\&A, 275, 101

Girardi, L., Bressan, A., Bertelli, G., \& Chiosi, C. 2000, A\&AS, 141, 371

Gray, D. F. 1988, Lectures on Spectral-line Analysis: F, G, and K Stars (Arva, Ontario: Publisher)

Guillot, T., Santos, N. C., Pont, F., et al. 2006, A\&A, 453, L21

Gonzalez, G. 2009, MNRAS, 399, L103

Hartman, J., Bakos, G., Torres, G., et al. 2009, ApJ, 706, 785

Haywood, M. 2008, A\&A, 482, 673

Hebb, L., Collier-Cameron, A., Triaud, A. H. M. J., et al. 2009, ApJ, 693, 1920

Hellier, C., Anderson, D. R., Gillon, M., et al. 2008, ApJ, 690, L89

Hellier, C., Anderson, D. R., Collier Cameron, A., et al. 2010, ApJ, submitted

Mayor, M., Pepe, F., Queloz, D., et al. 2003, Messenger, 114, 20

Morrison, H. L., Flynn, C., \& Freeman, K. C. 1990, AJ, 100, 1191

Munari, U., \& Zwitter, T. 1997, A\&A, 318, 269

Neves, V., Santos, N. C., Sousa, S. G., et al. 2009, A\&A, 497, 563

Pepe, F., Mayor, M., Galland, F., et al. 2002, A\&A, 388, 632

Perruchot, S., Kohler, D., Bouchy, F., et al. 2008, in Ground-based and Airborn Instrumentation for Astronomy II, ed. I. S. McLean, \& M. M. Casali, Proc. SPIE, 7014, 70140J

Pollacco, D. L., Skillen, I., Cameron, A. C., et al. 2006, PASP, 118, 1407

Pollacco, D., Skillen, I., Collier Cameron, A., et al. 2008, MNRAS, 385, 1576

Queloz, D., Mayor, M., Webber, L., et al. 2000, A\&A, 354, 99

Reid, I. N., Turner, E. L., Turnbull, M. C., et al. 2007, ApJ, 665, 767

Robin, A. C., Reylé, C., Derrière, S., \& Picaud, S. 2003, A\&A, 409, 523

Sato, B., Fischer, D., Henry, G., et al. 2005, ApJ, 633, 465

Stempels, H. C., Collier Cameron, A., Hebb, L., et al. 2007, MNRAS, 379, 773

Torres, G., Andersen, J., \& Gimenez, A. 2010, A\&ARv, 18, 67

Valenti, J. A., \& Piskunov, N. 1996, A\&AS, 118, 595

Valenti, J. A., \& Fisher, D. 2005, ApJS, 159, 141

Zacharias, N., Monet, D. G., Levine, S. E., et al. 2004, BAAS, 36, 1418 